\title{
SOME HAUSDORFF MATRICES NOT OF TYPE M
}

\author{
B. E. RHOADES ${ }^{1}$
}

Let $A=\left(a_{n k}\right)$ denote an infinite matrix. Then $A$ is said to be of type $\mathrm{M}$ if the conditions

$$
\sum_{n=0}^{\infty}\left|\alpha_{n}\right|<\infty, \quad \sum_{n=0}^{\infty} \alpha_{n} a_{n k}=0 \quad(k=0,1,2, \cdots)
$$

always imply $\alpha_{n}=0(n=0,1,2, \cdots)$.

Matrices of type $M$ were first introduced by Mazur [3] and sonamed by Hill [2]. In [2], Hill developed several sufficient conditions for a Hausdorff matrix to be of type M. He showed that there exists a regular Hausdorff matrix not of type $M$. The particular matrix used contained a zero on the main diagonal. He also posed the following question: Does there exist a regular Hausdorff matrix which has no zero elements on the main diagonal and which is not of type $M$ ? The purpose of this note is to answer the above question in the affirmative and establish several other related theorems.

A matrix $A=\left(a_{n k}\right)$ is called triangular if $a_{n k}=0$ for all $k>n$, and is called a triangle if $A$ is triangular and $a_{n n} \neq 0$ for each $n$. (Some authors use the word normal instead of triangle.) Throughout this paper all matrices and sequences contain real entries.

If we use the words finite sequence to describe a sequence containing only a finite number of nonzero terms, it is clear that a triangular matrix which is not a triangle cannot be of type $M$, since a finite sequence can be found satisfying (1). Also, if a matrix is a triangle, there can be no finite sequence as a solution of (1). Hill's example is a triangular matrix, not a triangle.

Let $\mu=\left\{\mu_{k}\right\}$ be a sequence, $\Delta$ a forward difference operator defined by $\Delta \mu_{k}=\mu_{k}-\mu_{k+1}, \Delta^{n} \mu_{k}=\Delta\left(\Delta^{n-1} \mu_{k}\right), n, k=0,1,2, \cdots$. Then a Hausdorff matrix $H=\left(h_{n k}\right)$ is written in the form $h_{n k}=C_{n, k} \Delta^{n-k} \mu_{k}$ for $k \leqq n$, and $h_{n k}=0$ for $k>n$. The sequence $\mu$ is called the generating sequence for the matrix $H$, and, for a regular matrix, we have the representation

$$
\mu_{n}=\int_{0}^{1} u^{n} d q(u) \quad(n=0,1,2, \cdots)
$$

Received by the editors September 8, 1962 and, in revised form, February 25, 1963.

${ }^{1}$ Work performed under the auspices of the U. S. Atomic Energy Commission. 
where $q(u)$ is a function of bounded variation on $0 \leqq u \leqq 1, q(0+)$ $=q(0)=0, q(1)=1$, and $q(u)=[q(u+0)+q(u-0)] / 2$ for $0<u<1$. For other properties of Hausdorff matrices see $[1, \mathrm{XI}]$. The function $q(u)$ is commonly referred to as the mass function for $\mu$.

In [2] it has been shown that, for regular Hausdorff matrices, (1) can be written in the form

$$
\int_{0}^{1} g_{k}(u) d q(u)=0 \quad(k=0,1,2, \cdots),
$$

where

$$
g_{k}(u)=\sum_{n=k}^{\infty} \alpha_{n} C_{n, k} u^{k}(1-u)^{n-k} \quad(k=0,1,2, \cdots) .
$$

Each of the functions $g_{k}(u)$ represents an absolutely and uniformly convergent series on $0 \leqq u \leqq 1$, and, for every $k$,

$$
g_{k}(u)=(-1)^{k} u^{k} g_{0}^{(k)}(u) / k !
$$

Theorem 1. Let

$$
\mu_{n}=\frac{b(n-a)}{(-a)(n+b)}, \quad a>0, b>0 \quad(n=0,1,2, \cdots) .
$$

Then the corresponding regular Hausdorff matrix is not of type $\mathrm{M}$.

Proof. If $a$ is a positive integer, then $H$ is not of type $\mathrm{M}$ as remarked above, since it has a zero on its diagonal.

Assume $a$ is not a positive integer. We may write $\mu_{n}$ in the form

$$
\mu_{n}=-\frac{b}{a}+\frac{(a+b)}{a}\left(\frac{b}{n+b}\right),
$$

and the corresponding mass function is

$$
q(u)= \begin{cases}1, & u=1, \\ (a+b) u^{b} / a, & 0 \leqq u<1 .\end{cases}
$$

Choose $\alpha_{0}=1, \alpha_{n}=(-1)^{n} a(a-1) \cdots(a-n+1) / n !, n \geqq 1$. Then $\sum_{n=0}^{\infty}\left|\alpha_{n}\right|<\infty$, and from (3) $g_{0}(u)=\sum_{n=0}^{\infty} \alpha_{n}(1-u)^{n}=u^{a}$. Using (4), we obtain $g_{k}(u)=(-1)^{k} a(a-1) \cdots(a-k+1) u^{a} / k !$, and the $g_{k}(u)$ satisfy (2). Therefore $H$ is not of type M.

The concept of type $M$ is of value only for reversible matrices, in particular, triangles. In general, it is superseded by the concept perfect; namely, that convergent sequences lie densely in the set of 
sequences transformed into convergent sequences by the matrix. For reversible matrices perfect and type $M$ are equivalent.

Since Hill's example is not reversible, it is reasonable to ask if it is perfect. The Hausdorff matrix in the example has moment generating sequence

$$
\mu_{n}=\frac{6(n-1)^{2}}{(n+1)(n+2)(n+3)}, \quad n=0,1,2, \cdots,
$$

with corresponding mass function $Q(u)=16 u^{3}-27 u^{2}+12 u, 0 \leqq u \leqq 1$. Instead of the corresponding Hausdorff matrix $H$, consider the matrix $K$, which agrees with $H$ except that $k_{11}=1$. Then $H$ and $K$ have the same convergence domains. Since $K$ is a triangle, $K$ is perfect if and only if it is of type M. Furthermore, if one chooses $\alpha_{0}=\alpha_{1}=0$, $\alpha_{n}=1 / n(n-1)$ for $n \geqq 2$, then equations (3) and (4) are applicable, and

$$
g_{0}(u)=\sum_{n=2}^{\infty} \frac{(1-u)^{n}}{n(n-1)} .
$$

Term by term differentiation leads us to

$$
g_{0}^{\prime \prime}(u)=\sum_{n=2}^{\infty}(1-u)^{n-2}=\frac{1}{u} .
$$

Noting that $g_{0}^{\prime}(1)=g_{0}(1)=0$, we obtain $g_{0}(u)=u(\log u-1)$. Using (3), the conditions in (2) become

$$
\begin{array}{r}
\int_{0}^{1} u d Q=0 \\
\int_{0}^{1} u \log u d Q=0
\end{array}
$$

and

$$
\int_{0}^{1}(u \log u-u) d Q=0
$$

It is easy to show that the first two are satisfied. The third condition is automatically satisfied, since it is a linear combination of the first two.

Therefore $K$ is not of type $\mathrm{M}$, and $H$ is not perfect.

If one examines the sequences of Theorem 1 for $a$ an integer, then one notes that the corresponding matrices are not reversible. It remains to determine if each such matrix is perfect. 
THEOREM 2. Let

$$
\mu_{n}=\frac{b(n-r)}{(-r)(n+b)}, \quad a>0, r a \text { positive integer }(n=0,1,2, \cdots) .
$$

Then the corresponding regular Hausdorf matrix is not perfect.

Proof. The technique will be the same as that of the preceding example; that is, let $K$ be $H$ with $k_{r r}=1$. It remains to show that $K$ is not of type M. For all $k>r$, equations (3) and (4) are valid. We wish to determine a sequence $\alpha$ which will satisfy (2). Using (5), (2) becomes

$0=\int_{0}^{1} g_{k}(u) d q=\frac{b}{r}\left[-\alpha_{k}+(r+b) \sum_{n=k}^{\infty} \alpha_{n} \frac{\Gamma(n+1) \Gamma(k+b)}{\Gamma(k+1) \Gamma(n+b+1)}\right]$,

for $k=r+1, r+2, \cdots$. Solving the above system for the $\alpha_{k}$ 's we obtain the recursion formula

$$
\alpha_{k+1}=\frac{(k-r) \alpha_{k}}{k+1}
$$

which gives us

$$
\alpha_{r+m+1}=\frac{m ! \alpha_{r+1}}{(r+2)(r+3) \cdots(r+m+1)}, \quad m=1,2,3, \cdots .
$$

Selecting $\alpha_{r+1}=1$, we can show that $\sum_{m=1}^{\infty}\left|\alpha_{r+m+1}\right|<\infty$. Substituting in (3) we obtain $g_{r+1}(u)=u^{r}$. Using (4) leads to

$$
g_{0}^{(r+1)}(u)=\frac{(-1)^{r+1}(r+1) !}{u}
$$

and hence that

$$
g_{k}(u)=\frac{u^{r}}{\left(\begin{array}{c}
k \\
m-1
\end{array}\right)}, \quad k=r+1, r+2, \cdots .
$$

Thus conditions (2) reduce to showing that

$$
\int_{0}^{1} u^{r} d q=0
$$

a condition easily verified.

There now remains the problem of determining the values $\alpha_{0}$ through $\alpha_{r}$. Because $H$ has been modified in row $r$, it is not possible 
to use (6) to obtain $g_{0}^{(r)}(u)$ and hence $g_{0}(u)$. However, one can use (1) to determine $\alpha_{r}$. Continued use of (1) will determine the remaining values $\alpha_{r-1}, \cdots, \alpha_{0}$. Thus $K$ is not of type $\mathrm{M}$, and $H$ is not perfect.

The following is an open question. Does there exist a regular Hausdorff matrix which is perfect, but not of type $M$ ?

I wish to thank the referee for his helpful suggestions.

\section{REFERENCES}

1. G. H. Hardy, Divergent series, Oxford, at the Clarendon Press, 1949.

2. J. D. Hill, On perfect methods of summability, Duke Math. J. 3 (1937), 702-704.

3. S. Mazur, Eine Anwendung der Theorie der Operationen bei Untersuchung der Toeplitzschen Limitierungsverfahren, Studia Math. 2 (1930), 40-50.

Argonne National Laboratory and

LAFAyette College 